\title{
Review of: "Impact of the COVID-19 pandemic on the practice of endocrine surgery"
}

\author{
Ralitza Parina ${ }^{1}$, Mohd Raashid Sheikh ${ }^{1}$ \\ 1 University of Southern California
}

Potential competing interests: The author(s) declared that no potential competing interests exist.

It is undoubtable that the COVID pandemic has had an abrupt and lasting impact on the practice patterns of surgeons. In their excellent study, Beninato et al invited members of the American Association of Endocrine Surgeons (AAES) to respond to a survey on the impact that the coronavirus pandemic has had on their professional, educational and clinical practice. In short, they found that a period of suspension of elective surgery was universal, there was a decrease in clinical volume, transition to virtual platforms for clinical and educational purposes and a trend toward increase in same-day discharge and decrease in postoperative observation in certain patients.

We appreciate this opportunity to highlight, expand on and critically appraise a few of their findings.

One of the premises of the study is the comparison of clinical variables "pre-COVID" and "postCOVID". Respondents of the survey, which was sent in September 2020, were allowed to interpret these time periods freely. As COVID-related restrictions on clinical care are about to start their $20^{\text {th }}$ month, referring to a "post-COVID" clinical practice seems like an oversimplification of the varied impact COVID has had over time. Most hospitals have gone through multiple iterations of restrictions and regulations on elective surgery and an increasing number of hospitals face critical staff shortages as a result of the pandemic. While the study highlights important changes in the delivery of surgical care, it appears to be largely reflective of the beginning months of the pandemic, while the longer term effects of COVID remain to be seen.

A finding we would like to explore further due to its potential applicability to other specialties is the trend toward increase in same-day discharges and decrease in postoperative observation length in total thyroidectomy patients. The authors also highlight several other studies which have described pathways for same-day discharge developed during the pandemic - after mastectomy with immediate reconstruction, transcatheter aortic valve replacement and robotic urologic procedures. As surgical techniques have improved, minimally invasive approaches become more commonplace and enhanced recovery after surgery pathways more prevalent, there has been a growing trend to decreasing postoperative length of stay and same-day discharge after a growing number of operations. The unique environment of COVID including high hospital censuses, restrictions on elective surgery, staff shortages and attempts at minimizing patient exposure have likely contributed to acceleration of this trend. While the trend was minimal in the present study, endocrine surgery may not be the most powerful example as outpatient 
endocrine surgery was an already well-established practice for many surgeons before the pandemic. Further inquiry into impact of COVID-era clinical changes on the prevalence of outpatient surgery and inpatient length of stay in other fields of surgery would be informative.

Another key finding in the study is the sharp increase in the use of telemedicine for clinical visits and virtual platforms for conferences and meetings. The authors suggest that the majority of survey responders felt that these platforms would continue post-pandemic and we similarly postulate that this will be one of the largest lasting impacts the pandemic will have on the practice of medicine. From a nonclinical perspective, the normalization of web-based platforms could lead to increased collaboration on research and minimize barriers to attendance of professional meetings.

From a clinical standpoint, as patients and providers alike have become more accustomed to webbased platforms and how to best utilize them, some advantages are often cited. Key among them is ease of access, minimizing barriers to closer preoperative counseling and postoperative follow-up. However, the impact of the rise of telehealth visits on patient's clinical outcomes as well as on patient satisfaction remains unstudied. Similarly, while the authors quantify a decrease in the number of office based procedures during the post-COVID era, the ultimate impact - if any - that these delays may have had on patient outcomes, including delays in diagnosis and operative interventions, remains out of the scope of the present methodology.

Overall, the study provides multiple starting points for discussion on the impact that COVID has had on the delivery of surgical care. While inherently limited by the use of a survey methodology which highlights the providers' over the patients' perspective and a somewhat nebulous definition of the "postCOVID" era, it starts to quantify the impact the pandemic has had on surgical care, and opens the door for future inquiries on the delivery and outcomes of care in a wider range of subspecialties. 